\title{
Initial Flow Matching Results of MHD Energy Bypass on a Supersonic Turbojet Engine using the Numerical Propulsion System Simulation (NPSS) Environment
}

\author{
Theresa L. Benyo ${ }^{1}$ \\ NASA Glenn Research Center, Cleveland, OH, 44135
}

\begin{abstract}
Preliminary flow matching has been demonstrated for a MHD energy bypass system on a supersonic turbojet engine. The Numerical Propulsion System Simulation (NPSS) environment was used to perform a thermodynamic cycle analysis to properly match the flows from an inlet to a MHD generator and from the exit of a supersonic turbojet to a MHD accelerator. Working with various operating conditions such as the enthalpy extraction ratio and isentropic efficiency of the MHD generator and MHD accelerator, interfacing studies were conducted between the pre-ionizers, the MHD generator, the turbojet engine, and the MHD accelerator. This paper briefly describes the NPSS environment used in this analysis and describes the NPSS analysis of a supersonic turbojet engine with a MHD generator/accelerator energy bypass system. Results from this study have shown that using MHD energy bypass in the flow path of a supersonic turbojet engine increases the useful Mach number operating range from 0 to 3.0 Mach (not using MHD) to an explored and desired range of 0 to 7.0 Mach.
\end{abstract}

\section{Nomenclature}

$\gamma \quad=$ specific heat ratio for air

$\eta_{N(g)}=$ enthalpy extraction ratio of the MHD generator

$\eta_{s(a)} \quad=$ isentropic efficiency for the MHD accelerator

$\eta_{s(g)} \quad=$ isentropic efficiency for the MHD generator

$\pi_{a} \quad=$ stagnation pressure ratio of the MHD accelerator

$\pi_{g} \quad=$ stagnation pressure ratio of the MHD generator

$\pi_{p} \quad=$ stagnation pressure ratio of the pre-ionizer

$\sigma \quad=$ electrical conductivity

$\chi=$ fraction of generator power diverted to pre-ionizer

$A_{g} \quad=$ cross sectional area of the MHD generator

$B \quad=$ magnetic field intensity

$C_{p} \quad=$ constant pressure specific heat

$K=$ Faraday loading parameter

$L \quad=$ length of the MHD generator

$\dot{m}_{a} \quad=$ mass flow rate of the air at the entry of the MHD generator

$M_{6} \quad=$ Mach number at the exit of the MHD accelerator

$M_{\text {entrance }}=$ Mach number at the entrance of a component

$M_{\text {exit }} \quad=$ Mach number at the exit of a component

$P_{0,6}=$ total pressure at the exit of the MHD accelerator

$P_{6} \quad=$ static pressure at the exit of the MHD acclerator

$P_{\text {elec }} \quad=$ power output of the MHD generator

$P_{\text {elecA }}=$ power input into the MHD accelerator

$T_{0,15}=$ stagnation temperature at the entrance of the MHD generator

$T_{0,2}=$ stagnation temperature at the exit of the MHD generator

$T_{0,53}=$ stagnation temperature at the entrance of the MHD accelerator

${ }^{1}$ Aerospace Engineer, Aeropropulsion Division, 21000 Brookpark Rd. MS 5-11, AIAA Senior Member. 
$T_{0,6} \quad=$ stagnation temperature at the exit of the MHD accelerator

$T_{0, \text { entrance }}=$ stagnation temperature at the entrance of a component

$T_{0, \text { exit }}=$ stagnation temperature at the exit of a component

\section{Introduction}

$\mathrm{T}$ The MHD energy bypass system has been shown ${ }^{1}$ to provide benefits for an existing aeropropulsion system. Shown schematically in Figure 1 is a MHD driven energy bypass engine using a turbojet. The MHD generator may be employed in the inlet, nozzle, and another duct, individually and in combination. The possibility of electromagnetically extracting part of the turbojet inlet air kinetic energy is the key feature. The concept potentially offers variable inlet geometry performance without the complexity of moving inlet parts.

Three primary aeropropulsion purposes are served by the concept. Firstly, flow enthalpy into the combustor is reduced allowing more efficient addition of energy in the combustor without exceeding temperature limitations on the turbine materials. Secondly, the applied electromagnetic fields and their body forces can enhance off-design performance by manipulating the flow features in the supersonic/hypersonic inlets thereby reducing total pressure losses and entropy changes for the same level of flow compression by other means. Thirdly, electrical power removed can be used for various on-board vehicle requirements including plasma flow control around the vehicle. In addition, the expanding flow in the high-speed nozzle may also be augmented by electromagnetic forces to generate more thrust. In order to achieve this interaction, the air needs to be ionized by an external means even up to fairly high flight speeds, and the leading candidates may be classified as electrical discharge devices.

The generation of energy from the engine gaseous working fluid involves conversion of the thermal and kinetic energy of the fluid, and, therefore, gives rise to a change in both the gas temperature and pressure, in addition to velocity (and Mach number). Considering the possibility of subsonic and supersonic flow speeds along the gas flowpath in different cases and locations, the geometry of the flowpath becomes a significant parameter in the design and performance estimation of the generator. These considerations obviously also arise during input of energy back to the working fluid, whether to accelerate it and derive thrust output, or simply to add thermal energy to it.

The work performed with this analysis will contribute to the following goals:

1. Cycle Analysis - Conduct cycle analyses to establish the operating conditions for a jet engine cycle that are optimal for kinetic energy transfer from inlet air to a downstream location in the engine.

2. Flowpath Design - The geometry of a flowpath operating from Mach 0 to 7 is to be determined. The resulting engine thrust to weight ratio and thermodynamic efficiency are to be determined using electrical conductivity, magnet mass, and MHD energy conversion as some of the parameters.

Flow matching among the MHD components and a turbojet engine is needed to help determine the flowpath design and appropriate operating conditions necessary for successful operation of MHD energy bypass for a supersonic turbojet engine. Flow matching means that the flow exiting one element of a turbojet engine matches the flow entering the adjacent element of the same engine. The Numerical Propulsion System Simulation (NPSS) ${ }^{2}$ environment can realistically model the physical interactions that take place throughout an aeropropulsion engine, accelerating the concept-to-production development time and reducing the need for expensive full-scale tests and experiments. Thus, the NPSS environment can achieve the flow matching needed to demonstrate that MHD energy bypass is beneficial for supersonic turbojet engines. The NPSS architecture supports implementing one-, two-, and three-dimensional models of the engine flow field and structure thus accommodating many fidelities of analysis. 


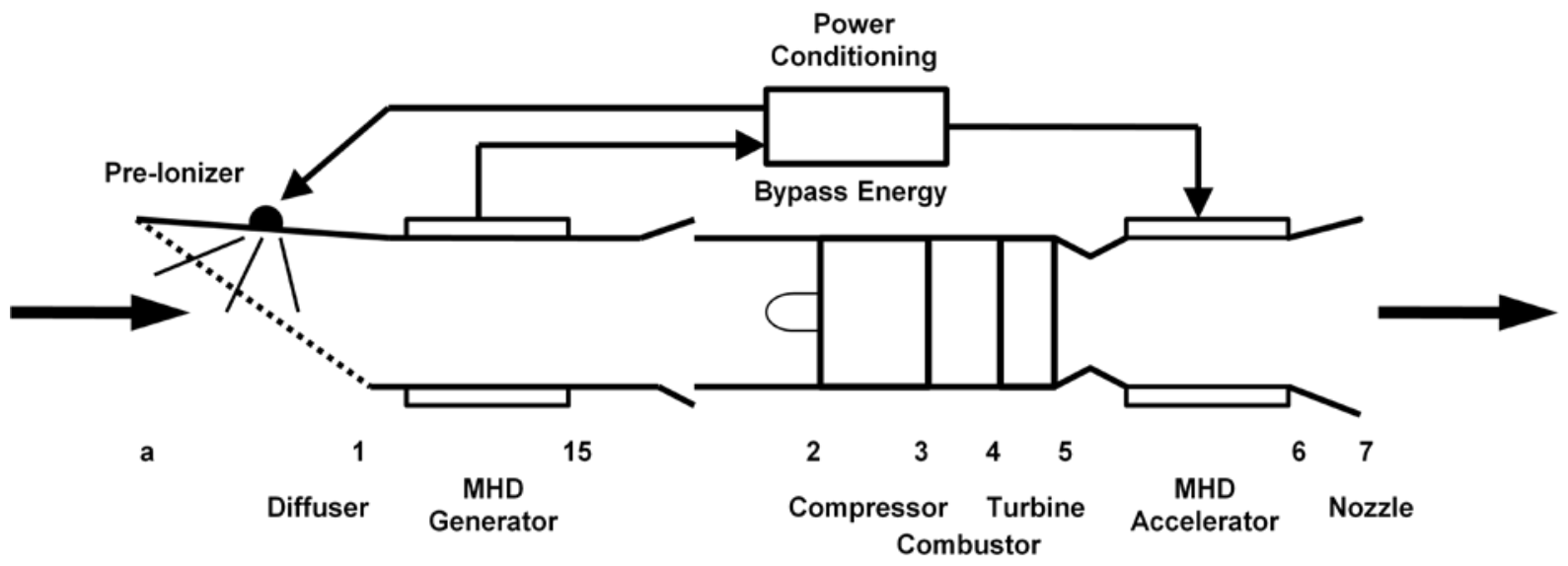

Figure 1. Schematic of MHD Controlled Turbojet for High Speed Propulsion

\section{Background}

\section{A. MHD Energy Bypass}

The MHD energy bypass system incorporated into a conventional gas turbine technology is a revolutionary concept which offers 3 distinct advantages. It allows turbomachinery to operate continuously over the entire range of Mach 0 to 7 where no deadweight engines are carried aloft. This revolutionary concept only uses hydrocarbon fuel where the plasma in the weakly ionized flow may be used to reform the hydrocarbon fuel into hydrogen. And finally, there is high potential for increased specific impulse.

A MHD energy bypass system bypasses kinetic energy (in the form of electrical power) from the inlet stream and uses it downstream to generate more thrust or reduce drag on the vehicle. This energy bypass is accomplished by using weak ionization of the inlet stream by an external means and MHD interaction with the ionized gas. The key feature of the engine lies in the additional hardware surrounding the jet engine. There are three main components of a MHD energy bypass system; the pre-ionizer, the MHD generator and the MHD accelerator. Figure 1 depicts the MHD energy bypass system around a supersonic turbojet engine.

The pre-ionizer for the MHD generator ionizes the flow coming into the MHD generator. The resulting ionized flow in conjunction with an applied magnetic field provides the benefits of flow control. The flow is ionized by the high voltage nanosecond discharge method ${ }^{3}$ where high-voltage, short pulse duration, high pulse repetition rate discharges generate ionization in supersonic cold flows $\left(T_{0}=300 \mathrm{~K}\right)$. The pre-ionizer for the MHD accelerator is positioned downstream of the supersonic turbojet engine's nozzle and operates in a similar manner as the pre-ionizer for the MHD generator.

The MHD generator towards the front of the engine acts as a flow control device and provides power to the preionizers and MHD accelerator. The electromagnetic fields produced by the MHD generator are used to enable variable inlet flow control that is similar to that controlled by variable geometry. The MHD generator is a nonobstructing means of total temperature reduction that can be controlled by applied magnetic fields and load parameter adjustment. Previous published results ${ }^{4}$ concentrated on the performance of the MHD generator by varying the amount of electrical power that is extracted out of the ionized flow and bypassed to the pre-ionizers and the MHD accelerator. The ratio of electrical power generated by the MHD generator and the power of the incoming flow is called the enthalpy extraction ratio and is represented by the following equation:

$$
\eta_{N(g)}=\frac{P_{\text {elec }}}{\dot{m}_{a} C_{p} T_{0,15}}
$$

where $P_{\text {elec }}$ is the electrical power and is represented by the following equation:

$$
P_{\text {elec }}=\sigma u^{2} B^{2} K(1-K) A_{g} L
$$


where $\sigma$ is the conductivity of the ionized air, $u$ is the velocity of flow entering the MHD generator in $\mathrm{m} / \mathrm{s}$, B is the applied magnetic field in Tesla, $\mathrm{K}$ is the Faraday loading parameter (usually held at 0.5 ) for the generator, $\mathrm{A}_{\mathrm{g}}$ is the cross sectional area of the MHD generator in $\mathrm{m}^{2}$, and $\mathrm{L}$ is the length of the generator in meters. In that study it was shown that applying a magnetic field to a flow path in the Mach 2.0 to 3.5 range can increase the specific thrust of the turbojet engine up to as much as $420 \mathrm{~N} /(\mathrm{kg} / \mathrm{s})$ provided that the magnitude of the magnetic field is in the range of 1-5 Tesla.

Most of the electrical power generated by the MHD generator is bypassed to the MHD accelerator at the back of the engine. As a result, the MHD accelerator is able to accelerate the flow exiting the engine by applying electromagnetic forces to the ionized flow and works together to augment the thrust generated.

\section{B. Flow Matching with NPSS}

To achieve flow matching between the MHD components and a supersonic turbojet engine, a comprehensive thermodynamic cycle analysis environment is needed. The Numerical Propulsion System Simulation (NPSS) created at NASA Glenn Research Center is one such environment that can properly match the flows between all the elements (inlet, compressor, burner, turbine, nozzle, etc.) of an aeropropulsion system. NPSS is structured in a way that allows an object oriented approach of using computers and computer codes to simulate aeropropulsion systems. Due to the complex interactions between the engine components as well as large changes in environmental operating conditions, computer codes are required to predict the performance of all but the most simple, ideal engine. NPSS is one such computer code and its object-oriented nature enables nearly any conceivable engine architecture to be modeled accurately.

One such example of a turbojet engine architecture ${ }^{5}$ is illustrated in Figure 2. Each box in the figure represents a section of code that is written to model the physics that governs the operation of each element of an aeropropulsion system. For example, a duct element (Duct1 on Figure 2) contains computer code that performs a simple adiabatic pressure loss calculation and maintains constant enthalpy while the pressure loss is applied. The small circle connections between the boxes represent the flows out of one element and into an adjacent element (which need to be equal) thus performing a flow match. Each flow station is labeled above the element. For example, FS_1 labels the flow station at the exit of the inlet and the entrance of Duct1. The large circle dashed line connection between the high speed compressor (HPC) and the high speed turbine (HPT) represent the power transferred from the turbine to the compressor through a shaft (HP_shaft). The diamond connections indicate fuel flow as seen between the boxes labeled FuelIn and Burner. Finally, the box labeled PERF is an element that calculates overall engine performance results such as gross thrust and net thrust.

The NPSS environment is essentially textfile-based. The text is broken down into three main categories; creation of objects, assignment of values to variables, and commands. The following list of files illustrates an example of those required for successful operation within NPSS:

- turbojet.mdl - this file configures the engine and runs the simulation

- turbojet_HPC.map - this file contains the compressor performance map

- turbojet_HPT.map - this file contains the turbine performance map

- turbojet.view_page - this file determines the format of the output

- turbojet.output - this is the output file generated by running the simulation

The next section of the paper describes how the flow matching was achieved for a supersonic turbojet engine using a MHD energy bypass system. 


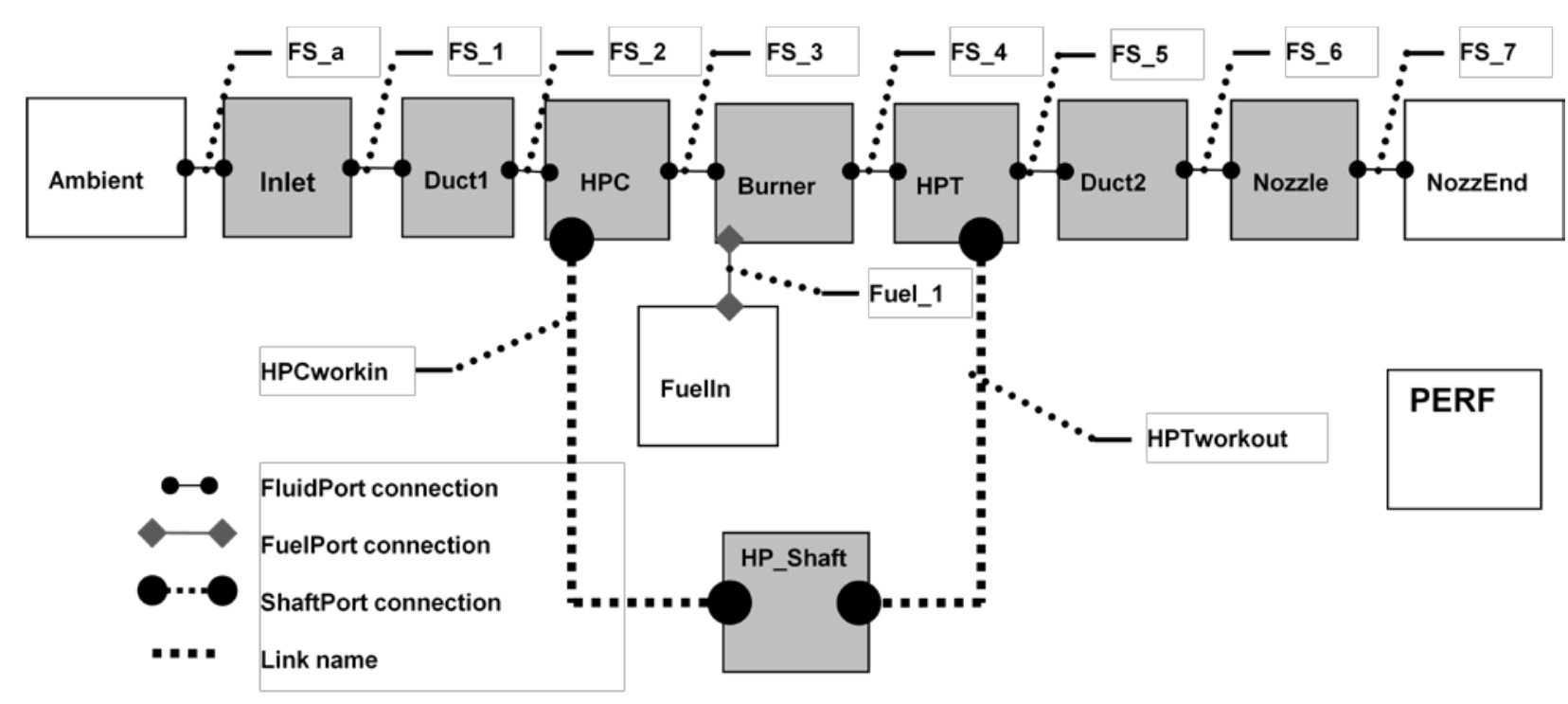

Figure 2. Diagram of a NPSS turbine engine model. Reference stations and process names are indicated

\section{Analysis Goals}

The goal of this analysis is to demonstrate flow matching between the MHD energy bypass system and a supersonic turbojet engine and to build upon previous work performed by Litchford ${ }^{6}$ et. al. and Benyo ${ }^{4}$. In order to achieve this goal, the NPSS environment is chosen since it can help further enhance a thermodynamic cycle analysis of a turbojet engine with a MHD generator and a MHD accelerator. This analysis uses a supersonic turbojet engine model previously developed by Christopher Snyder (NASA GRC) within the NPSS environment and builds a new NPSS object within that model called DuctMHD. The new NPSS object is computer code used for simulating the MHD energy bypass system; the pre-ionizer, the MHD generator and the MHD accelerator.

The DuctMHD object that was created with computer code contains all the equations necessary to model the physics of the components. The static pressure is held constant for each MHD component. Because of that condition, the exit Mach number of the pre-ionizers can be calculated with the following equation ${ }^{7}$ (Heiser, Eq. 2112),

$$
M_{\text {exit }}=\frac{M_{\text {entrance }}}{\sqrt{\frac{T_{0, \text { exit }}}{T_{0, \text { entrance }}}\left(1+\frac{\gamma-1}{2} M_{\text {entrance }}^{2}\right)-\frac{\gamma-1}{2} M_{\text {entrance }}^{2}}}
$$

where $T_{0, \text { exit }}$ is computed from the total temperature at the entrance using the enthalpy extraction ratio. As derived in Ref. 8, the enthalpy extraction/addition ratio is determined by the output power produced by the MHD generator and the enthalpy input through the 'inlet' of the MHD generator. For this analysis, we set that ratio as a percent of the energy being extracted. Therefore, the stagnation (total) temperature at the exit of the pre-ionizer is calculated as follows:

$$
T_{0, e x i t}=\frac{T_{0, \text { entrance }}}{1-\chi \eta_{N(g)}}
$$

In the traditional manner, the stagnation pressure ratio of the pre-ionizers is calculated as shown here ${ }^{7}$ (Heiser, Eq. 2-114).

$$
\pi_{p}=\left[1+\frac{\gamma-1}{2} M_{\text {entrance }}^{2}\left(1-\frac{T_{0, \text { entrance }}}{T_{0, \text { exit }}}\right)\right]^{\gamma / 1-\gamma}
$$


The MHD generator extracts kinetic energy from the incoming flow ionized by the pre-ionizer. The calculation uses the energy of the flow multiplied by the desired enthalpy extraction ratio as shown in the following equation ${ }^{8}$.

$$
P_{\text {elec }}=\dot{m}_{a} C_{p} T_{0,15} \eta_{N(g)}
$$

The exit temperature of the MHD generator also has to be computed using the enthalpy extraction ratio since energy is being taken out of the flow.

$$
T_{0,2}=T_{0,15}\left(1-\eta_{N(g)}\right)
$$

The stagnation pressure ratio for the MHD generator is

$$
\pi_{g}=\left(1-\frac{\eta_{N(g)}}{\eta_{s(g)}}\right)^{\frac{\gamma}{\gamma-1}}
$$

Because the MHD generator extracts energy, the MHD accelerator takes that energy and uses it to add more energy to the flow. Hence there is a temperature increase calculated as

$$
T_{0,6}=T_{0,53}+\frac{P_{\text {elec } A}}{\dot{m} C_{p}}
$$

where $P_{\text {elecA }}$ is the energy used by the MHD accelerator that is bypassed from the MHD generator. The energy is a percentage of $\mathrm{P}_{\text {elec }}$ and is expressed as:

$$
P_{\text {elecA }}=P_{\text {elec }}(1-2 \chi)
$$

where for this analysis, $10 \%$ of the power that is bypassed is used for both pre-ionizers ( $5 \%$ of power is used for each. The stagnation pressure ratio for the MHD accelerator is expressed as

$$
\pi_{a}=\left[1+\eta_{s(a)} \frac{T_{0,6}-T_{0,53}}{T_{0,53}}\right]^{\frac{\gamma}{\gamma-1}}
$$

Since the process is isentropic, we can write the exit Mach number as the following ${ }^{9}$ (Hesse, 3.6)

$$
M_{6}=\sqrt{\frac{2}{\gamma-1}\left[\left(\frac{P_{0,6}}{P_{6}}\right)^{\frac{\gamma-1}{\gamma}}-1\right]}
$$

All of the above equations are part of the new element called DuctMHD that is coded within NPSS. The equations describe the physics of the MHD components; pre-ionizers, MHD generator and MHD accelerator. A test harness was written to test out the new NPSS element and it was found to behave as expected.

The provided supersonic turbojet engine model in the NPSS environment was first studied and runs were made without the additional MHD energy bypass system to obtain a baseline of results. Four additional components; the pre-ionizer for the flow entering the MHD generator, the MHD generator, the pre-ionizer for the flow entering the MHD accelerator and the MHD accelerator were developed as DuctMHD objects. The new schematic of NPSS objects for this new model is illustrated in Figure 3. The analysis was optimized by varying the enthalpy extraction ratio and the isentropic efficiency of the MHD generator and MHD accelerator for the desired operating range of 0.0 to 7.0 Mach for the supersonic turbojet engine with the MHD energy bypass system. 


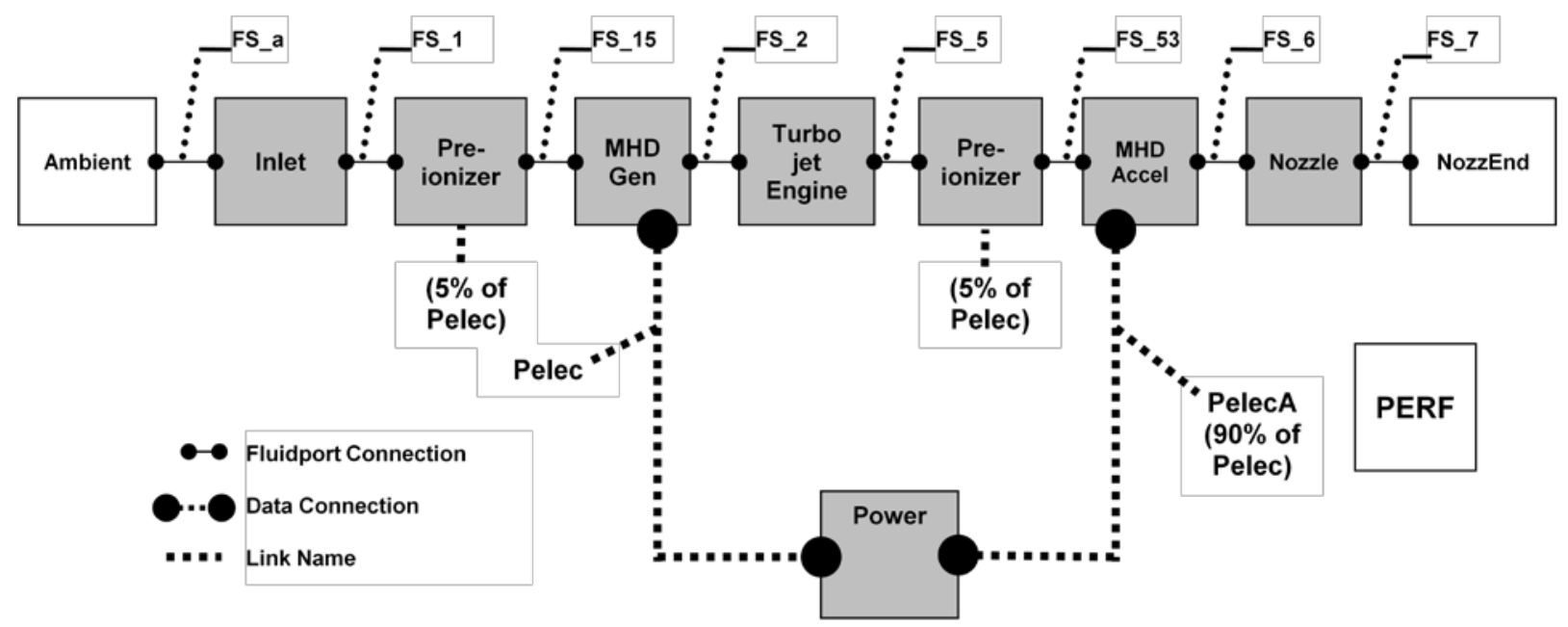

Figure 3. Schematic of MHD Energy Bypass with Turbojet Engine within NPSS. Reference stations and process names are indicated

In this study, the focus was on a supersonic turbojet engine somewhat like the Allison J-102 with a MHD energy bypass system and the following operating conditions ${ }^{1,10}$ :

\section{System}

Freestream temperature: $217 \mathrm{~K}$ to $238 \mathrm{~K}$

Turbojet engine length: about 1.2 meters

\section{Pre-ionizers}

Fraction of MHD generator power diverted to each pre-ionizer $(\chi): 0.05$

Conductivity: 1 mhos/m

\section{MHD Generator}

Mass flow rate of air $\left(m_{a}\right): 4.22 \mathrm{~kg} / \mathrm{s}$

Enthalpy extraction ratio $\left(\eta_{\mathrm{N}(\mathrm{g})}\right)$ : varies to optimize flow matching (ranges from 0.25 to 0.31 )

Isentropic efficiency: 0.9 and 0.98

Length: 10 meters

Cross sectional area: $0.5 \mathrm{~m}^{2}$

\section{MHD Accelerator}

Isentropic efficiency: 0.9 and 0.98

\section{Nozzle}

Exit velocity coefficient: 0.90

Throat discharge coefficient: 0.97 


\section{Results from Simulation}

A series of runs of the model described in the previous section was completed and verified that MHD technology can expand an existing turbojet engine's operating map. A group of operating parameters was studied that resulted from the runs in the NPSS environment.
a) Enthalpy
b) Mach number in flowpath
c) Total pressures
d) Overall net thrust
e) Overall gross thrust

The data of most interest will be presented here. The focus for this analysis is the energy that is bypassed from the MHD generator to the MHD accelerator (total enthalpy), the pressure/temperature changes throughout the flowpath of the supersonic turbojet engine and the net thrust generated with and without the MHD energy bypass system. The best results were obtained when the enthalpy extraction ratio was between 0.25 and 0.31 and the isentropic efficiency was $98 \%$ for both the MHD generator and MHD accelerator.

The Mach number in the flowpath is altered by using MHD energy bypass as evidenced in Figure 4. Without activating (extracting energy from the flow) the MHD components, the Mach number between FS_15 and FS_6 remains relatively constant. When the MHD components are activated, the flow slows down before entering the supersonic turbojet at FS_2 and enables subsonic flow through the turbojet. The slowdown is achieved by the MHD generator (between FS_15 and FS_2). After the flow through the turbojet, the flow speeds back up as the MHD accelerator (FS_53) uses the bypassed energy to inject more power into the flow (FS_6).

Likewise, the total temperature in the flow is altered by using MHD energy bypass. Figure 5 shows the total temperature at each flow station where no energy is extracted and bypassed onto the MHD accelerator. As expected, the total temperature increases rapidly from the exit of the MHD generator (FS_2) to the entrance of the pre-ionizer before the MHD accelerator (FS_53). When energy is extracted out of the flow at the MHD generator, Figure 6 shows the result. The main outcome to note here is that the rate of increase in total temperature from FS_2 to FS_5 is not as steep compared to when the MHD components are not activated. Also note that the preionizer for the MHD generator adds some heat to the flow. After FS_5, heat is also reintroduced into the flow field by the MHD accelerator's pre-ionizer and the MHD accelerator, causing higher total temperature at the nozzle (FS_7) of the engine.

Total pressure effects are shown in Figures 7-8. As expected, when the MHD components are activated there is a total pressure loss due to the enthalpy extraction at the MHD generator. Total pressure losses are greater than the non-activated case, but should not greatly affect the operation of the supersonic turbojet.

The net thrust and gross thrust outcomes are shown in Figures 9-10. For a mass flow of $4.22 \mathrm{~kg} / \mathrm{s}$, a net thrust range of $2500 \mathrm{~N}$ (at Mach 3.5) down to

\section{Mach Number of Flowpath with MHD and $\mathbf{9 8 \%}$ Isentropic Efficiency}

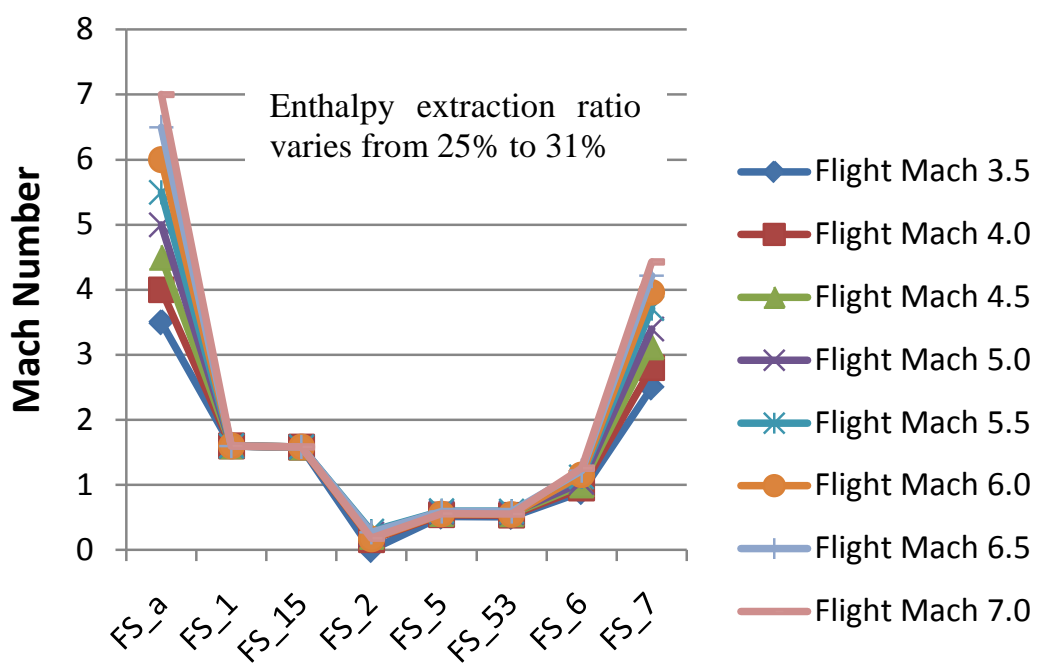

Flow Station

Figure 4. Mach Number vs. Flow Station. With MHD components activated. 
$800 \mathrm{~N}$ (at Mach 7.0) is obtained. Likewise, a gross thrust range of $4200 \mathrm{~N}$ (at Mach 3.5) up to $9700 \mathrm{~N}$ (at Mach 7.0) is obtained. These ranges are achieved with a 98\% isentropic efficiency for both the MHD generator and MHD accelerator. The enthalpy extraction ratio was found to be between $25 \%$ and $31 \%$ and varied for each calculated ambient (flight) Mach number. Using equations (1) and (2) of this paper, it was found that the applied magnetic field required for these results was around 3 Tesla which is consistent with previous work ${ }^{4}$.

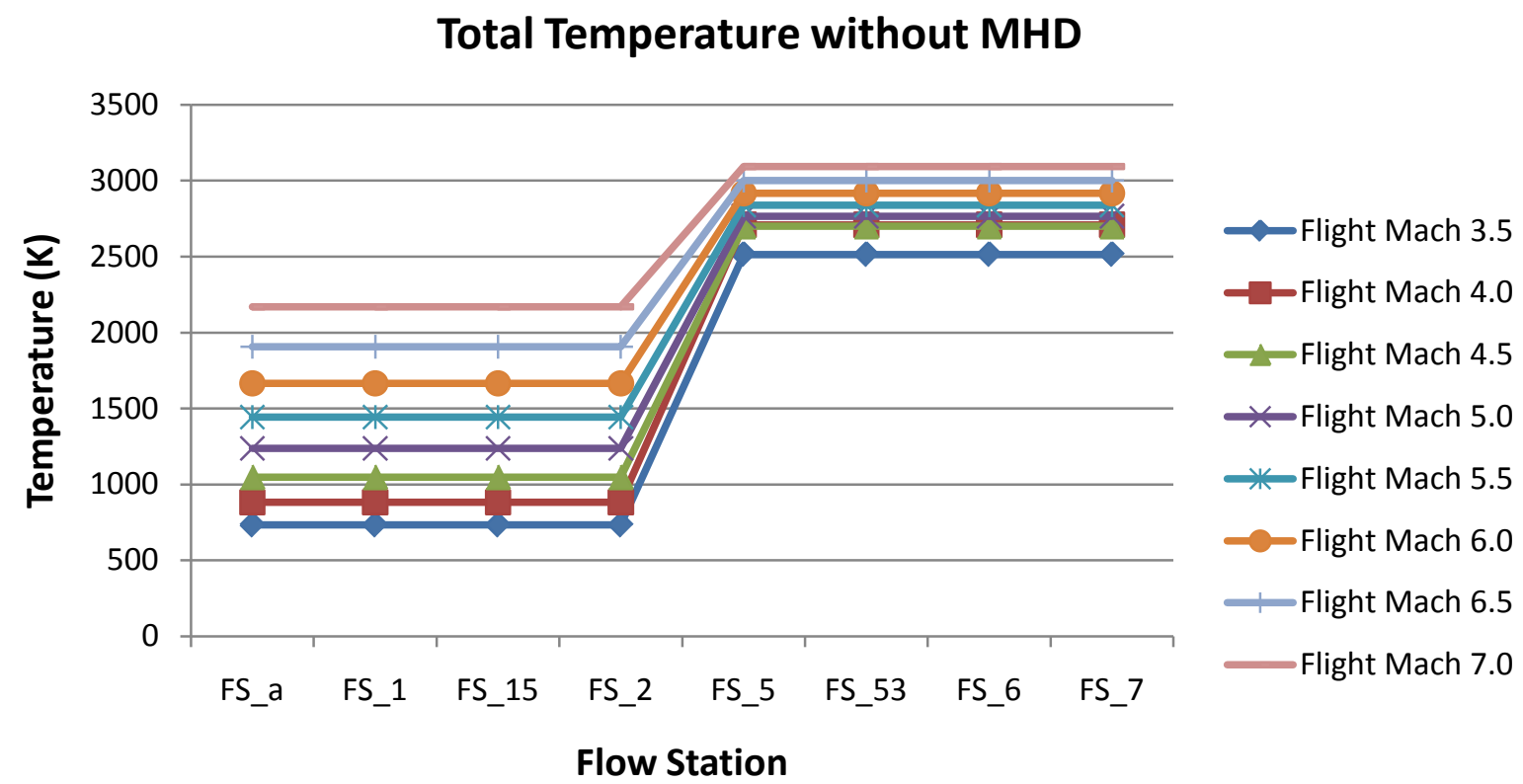

Figure 5. Total Temperature vs. Flow Station

\section{Total Temperature with MHD and 98\% Isentropic Efficiency}

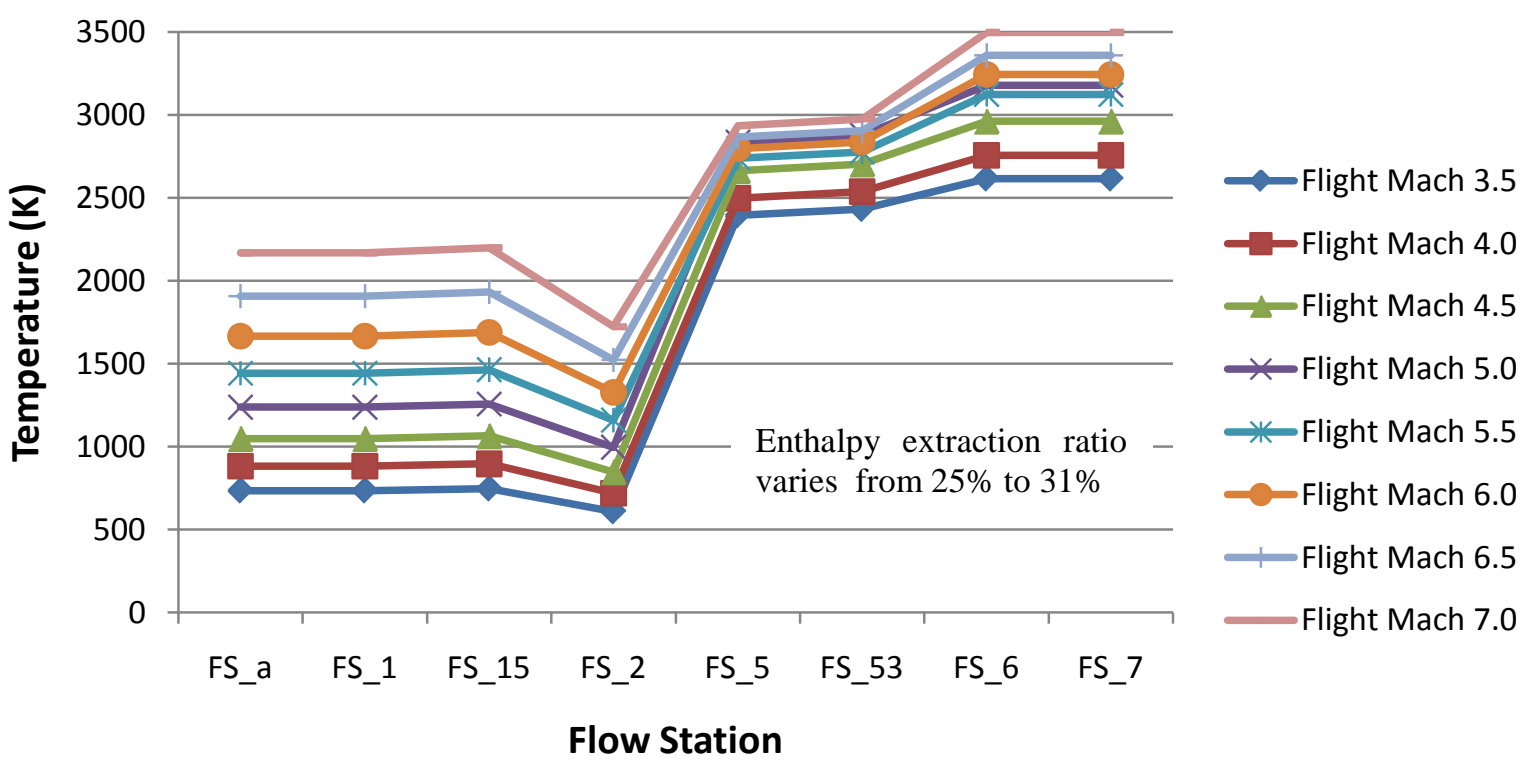

Figure 6. Total Temperature vs. Flow Station. With MHD components activated. 
Total Pressure without MHD

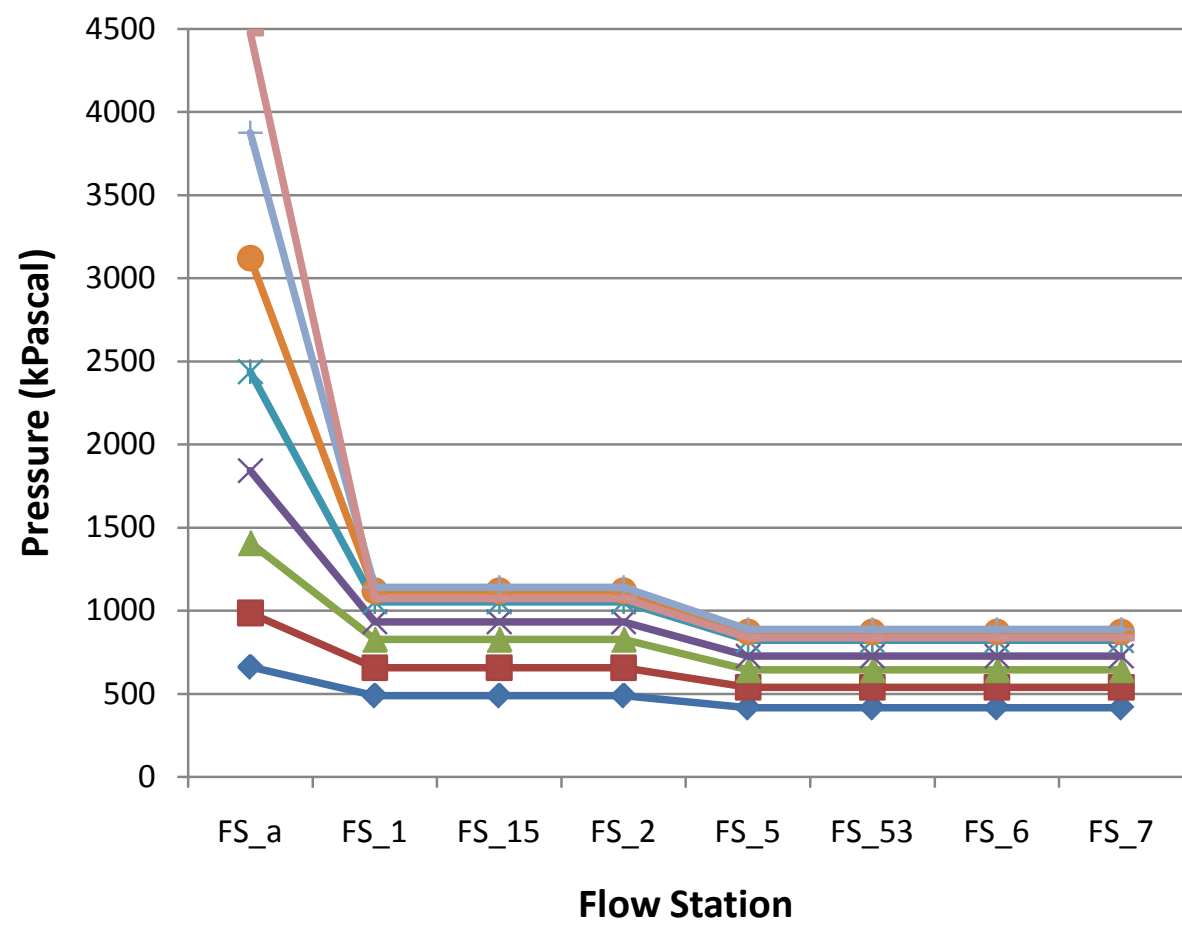

Flight Mach 3.5

Flight Mach 4.0

$\longrightarrow$ Flight Mach 4.5

Flight Mach 5.0

* Flight Mach 5.5

-Flight Mach 6.0

Flight Mach 6.5

Flight Mach 7.0

Figure 7. Total Pressure vs. Flow Station.

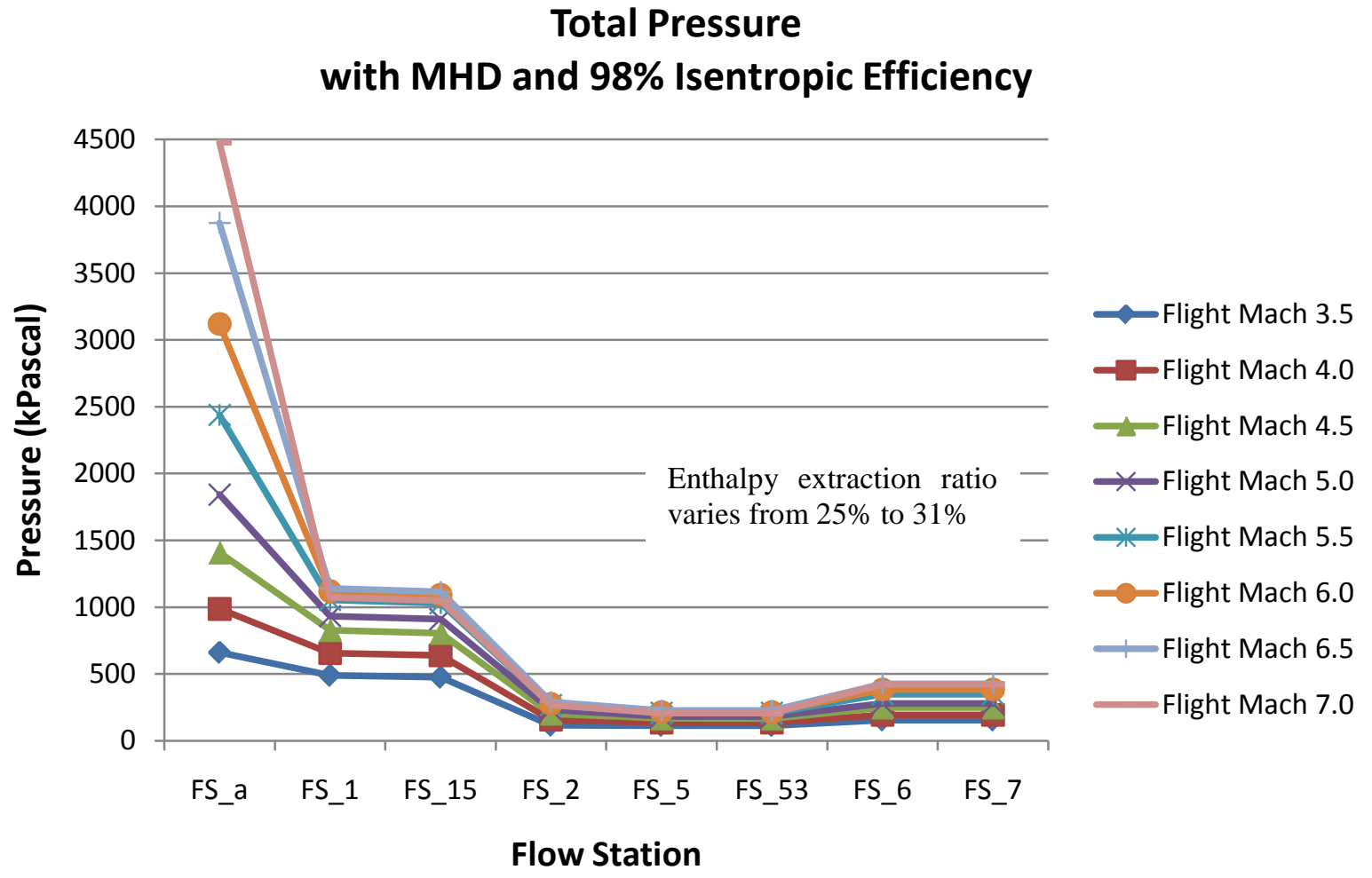

Figure 8. Total Pressure vs. Flow Station. With MHD components activated. 


\section{Supersonic Turbojet Performance}

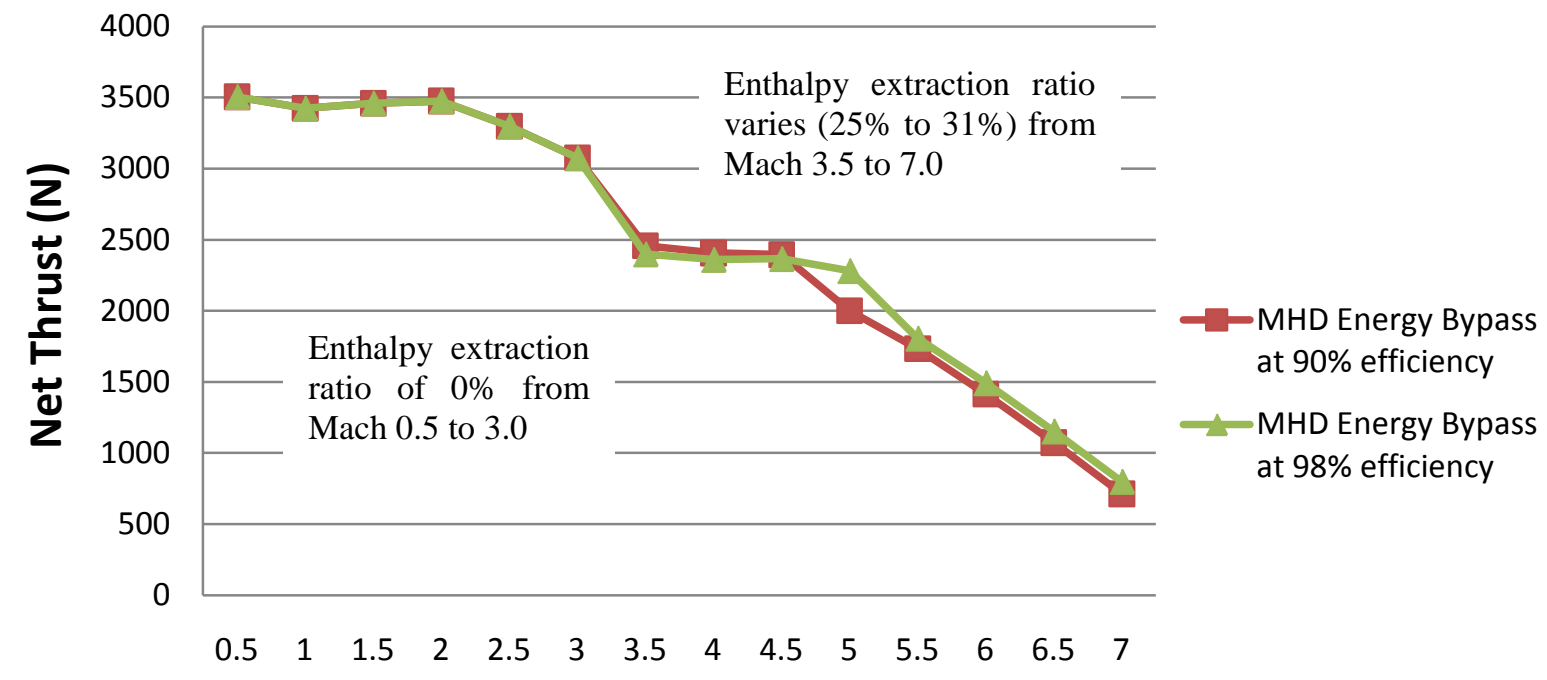

\section{Flight Mach Number}

Figure 9. Net Thrust vs. Flight Mach Number

\section{Supersonic Turbojet Performance}

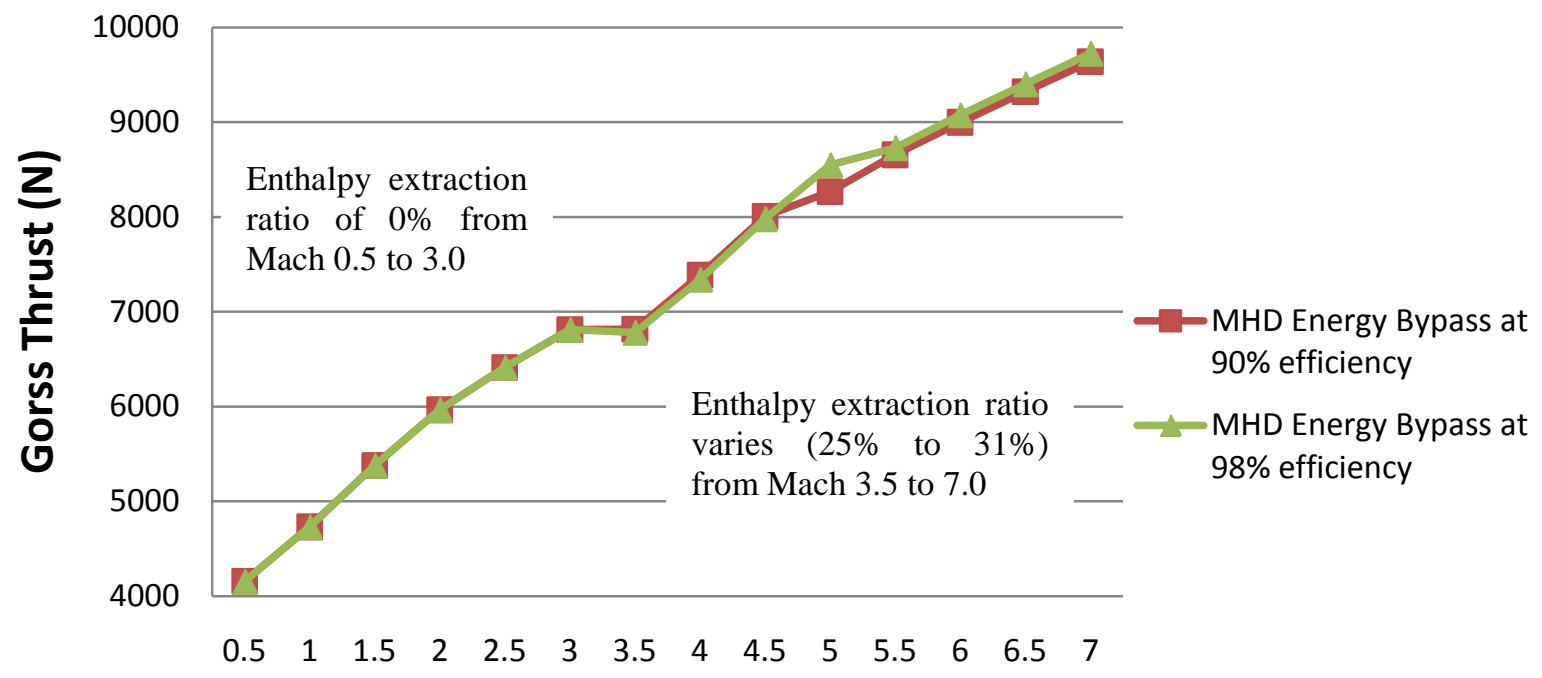

\section{Flight Mach Number}

Figure 10. Gross Thrust vs. Flight Mach Number 


\section{Conclusion}

Through the new analysis described here, it is shown that using MHD energy bypass in a supersonic turbojet engine will increase the engines operating range to a desired extended range of Mach 3.5 to Mach 7.0 into the hypersonic flight regime. All this is shown with a magnetic field of around 3 Tesla with an enthalpy extraction ratio between 0.25 and 0.31 , a conductivity level of $1 \mathrm{mhos} / \mathrm{m}$, a Faraday loading parameter of 0.5 , a MHD generator cross sectional area of $0.5 \mathrm{~m}^{2}$ and a MHD generator length of 10 meters.

MHD technology is a valuable addition to hypersonic flight in that it can expand the operating conditions of existing turbojet engines such as the supersonic turbojet engine studied in this analysis. This cycle analysis helps establish the operating conditions for a jet engine cycle with MHD energy bypass that are optimal for kinetic energy transfer from inlet air to a downstream location in the engine.

\section{A. Future Plans}

It is planned to establish a model for a jet engine with MHD energy bypass and determine the design and operating conditions in which the thrust-to-weight ratio, thrust per unit mass of fuel consumption, and effectiveness of energy utilization are maximized.

Specific future work in this area of full engine cycle analyses using MHD technology includes:

- Further refine the operating conditions for a turbo-jet engine cycle, that are optimal for kinetic energy transfer from inlet air (Mach reduction to 0.8 ) to a downstream location in the engine in a bypass mode.

- Continue flow matching studies with a more comprehensive NPSS model of a supersonic turbojet engine.

- Establish the interaction parameter and efficiency for MHD conversion of kinetic energy of the ionized gas to electrical energy by using environments such as Matlab and NPSS.

- Quasi1D MHD theory will be employed to conduct inlet analysis evaluations using experimentally determined conductivity, interaction parameters, and the mass flow captured at the inlet.

\section{Acknowledgments}

Theresa L. Benyo thanks Dr. Isaiah Blankson, Dr. Steve Schneider, Chris Snyder and Justin Gray of NASA Glenn Research Center for their guidance and encouragement throughout this research.

\section{References}

${ }^{1}$ Blankson, I.M., S. Schneider, "Hypersonic Engine using MHD Energy Bypass with a Conventional Turbojet”, presented at the 12th AIAA International Space Planes and Hypersonic Systems and Technologies, December 2003, AIAA $2003-6922$.

2“NPSS User Guide: Software Release: NPSS_1.6.5”, Numerical Propulsion System Simulation NASA Industry Cooperative Effort, March 12, 2008.

${ }^{3}$ Nishihara, M., J. W. Rich, W. R. Lempert, and I. V. Adamovich, "Low-temperature M=3 Flow Deceleration by Lorentz Force”, Phys. Fluids 18, 086101 (2006).

${ }^{4}$ Benyo, T.L., “The Effect of MHD Energy Bypass on Specific Thrust for Supersonic Turbojet Engine”, January 2010, AIAA-2010-0232.

${ }^{5}$ Jones, S. M., “An Introduction of Thermodynamic Performance Analysis of Aircraft Gas Turbine Engine Cycles Using the Numerical Propulsion System Simulation Code”, NASA TM-2007-214690, March 2007.

${ }^{6}$ Litchford, R.J., J.W. Cole, V.A. Bityurin, and J.T. Lineberry, “Thermodynamic Cycle Analysis of MagnetohydrodynamicBypass Hypersonic Airbreathing Engine”, NASA TP-2000-210387.

${ }^{7}$ Heiser, W. H. and D. T. Pratt with D. H. Daley and U. B. Mehta, Hypersonic Airbreathing Propulsion, AIAA Education Series, AIAA, Washington, D.C., 1994, pp. 77-81.

${ }^{8}$ Murthy, S.N.B and I.M. Blankson, “MHD Energy Bypass Turbojet-Based Engines”, presented at the $51^{\text {st }}$ International Astronautical Congress, October 2000, Rio de Janeiro, Brazil, IAF-00-5-5-05.

${ }^{9}$ Hesse, W. J. and N.V.S. Mumford, Jr., Jet Propulsion for Aerospace Applications, $2^{\text {nd }}$ ed., Pitman Publishing Corporation, New York, 1964, pp. 46-47.

${ }^{10} \mathrm{~J}-102$ packet includes engine specifications and engine performance envelope from Isaiah Blankson. 